\author{
Nucleon \\ James W Goodman \\ Louisiana State University \\ University Station \\ Baton Rouge, Louisiana
}

\begin{abstract}
The force here is the nuclear force binding the nuclei together. A magnetic dipole has the energy given by $-13.6^{\star} 2^{\star} n^{\wedge} 2[3]$. A particle with $n=822$ is a magnetic dipole with magnetic quantum number $m=1,2,3, \ldots$ so that $-18.3786 \mathrm{Mev}^{*} \mathrm{~m}$ is an energy/mass which we may associate with the nucleus. The relativistic correction 2[4] is required showing that the dipole velocity is near $\mathrm{c}$. The nuclei are bound with particles of mass $18 \mathrm{Mev}$ consisting of 6 magnetic dipoles each. The binding energy tracks very well with Z-1 particles where $\mathrm{Z}$ is the atomic number of the element. The particle is named a Nucleon after the manner of graviton and should not be confused with the common name for a nuclear particle (proton or neutron).
\end{abstract}

\title{
Keywords
}

Quantum Physics Energy States Hilbert Space Multibody Solution Magnetic Dipole Electron Mass Nuclear Binding

\section{Academic Discipline}

Physics

\section{Subject Classification}

Quantum Mechanics

\section{Approach}

Theoretical

\section{Introduction}

The material here presented uses the Texas Method of solving mathematical theorems. A few lemmas are required before the derivation of the solution of the strong force.

The force here is the nuclear force binding the nuclei together. A magnetic dipole has the energy given by $-13.6^{\star} 2^{\star} n^{\wedge} 2[3]$. A particle with $n=822$ is a magnetic dipole with magnetic quantum number $m=1,2,3, \ldots$ so that $-18.3786 \mathrm{Mev}^{*} \mathrm{~m}$ is an energy/mass which we may associate with the nucleus. The relativistic correction 2[4] is required showing that the dipole velocity is near $\mathrm{c}$.

\section{Derivation}

Hypothesis: There exists a particle called a nucleon that is a magnetic dipole with quantum number $n=822$ that gives the total binding energy of the helium nucleus in theory.[3]

Hypothesis: Each successive nuclear binding energy is given by the magnetic quantum number $m=1,2,3, \ldots[3]$ The helium nucleus has 4 particles in the nucleus and $n(n-1) / 2$ ways of binding. $4{ }^{\star} 3 / 2=6$. The magnetic energy quantum number is 137.6 ways * $137=822$. This gives the ground state energy of the helium as $-18.4 \mathrm{Mev}$ with the relativistic correction of 2 included. From [4] the energy is $28.3 \mathrm{Mev}$. However, the energy calculated for the lithium nucleus is -36.8 Mev which is only $7 \%$ lower than the -39.2 from [4]. Each dipole added forms a new tetrahedron of energy $-18.4 \mathrm{Mev}$. The table below gives the calculated values compared to the reference.

\section{Dialogue}

The table below gives energies in Mev. $Z$ is the atomic number, $m$ is the magnetic quantum number and where the reference energies have not yet been added a zero is given resulting in a division error which may be ignored. The \% variations for lithium to neon give credibility to the derivation and further calculations remind that the values in the reference are partly calculated also. Where the reference value is given farther down the table the atomic symbol is followed with XX.

\section{Results}

\author{
Nuclear Force
}




\begin{tabular}{|c|c|c|c|c|c|}
\hline & Z & $\mathrm{m}$ & $\mathrm{m}^{*} 18.37$ & REF Mev & $\%$ var \\
\hline D & 1 & 0 & 0 & -2.2 & \\
\hline $\mathrm{He}$ & 2 & 1 & -18.3786 & -28.3 & -36 \\
\hline $\mathrm{Li}$ & 3 & 2 & -36.75721 & -39.2 & -7 \\
\hline $\mathrm{Be}$ & 4 & 3 & -55.13581 & -58 & -5 \\
\hline B & 5 & 4 & -73.51442 & -76 & -4 \\
\hline C & 6 & 5 & -91.89302 & -92 & -1 \\
\hline $\mathrm{N}$ & 7 & 6 & -110.2716 & -104.7 & 5 \\
\hline O & 8 & 7 & -128.6502 & -128 & 0 \\
\hline$F$ & 9 & 8 & -147.0288 & -148 & -1 \\
\hline $\mathrm{Ne}$ & 10 & 9 & -165.4074 & -161 & 2 \\
\hline $\mathrm{Na}$ & 11 & 10 & -183.786 & 0 & \#DIV/0! \\
\hline $\mathrm{Mg}$ & 12 & 11 & -202.1647 & 0 & \#DIV/0! \\
\hline $\mathrm{Al}$ & 13 & 12 & -220.5433 & 0 & \#DIV/0! \\
\hline $\mathrm{Si}$ & 14 & 13 & -238.9219 & 0 & \#DIV/0! \\
\hline $\mathrm{P}$ & 15 & 14 & -257.3005 & 0 & \#DIV/0! \\
\hline$S$ & 16 & 15 & -275.6791 & 0 & \#DIV/0! \\
\hline Clxx & 17 & 16 & -294.0577 & -336 & -13 \\
\hline $\mathrm{Ar}$ & 18 & 17 & -312.4363 & 0 & \#DIV/0! \\
\hline $\mathrm{K}$ & 19 & 18 & -330.8149 & 0 & \#DIV/0! \\
\hline $\mathrm{Ca}$ & 20 & 19 & -349.1935 & 0 & \#DIV/0! \\
\hline Sc & 21 & 20 & -367.5721 & 0 & \#DIV/0! \\
\hline $\mathrm{Ti}$ & 22 & 21 & -385.9507 & 0 & \#DIV/0! \\
\hline V & 23 & 22 & -404.3293 & 0 & \#DIV/0! \\
\hline $\mathrm{Cr}$ & 24 & 23 & -422.7079 & 0 & \#DIV/0! \\
\hline $\mathrm{Mn}$ & 25 & 24 & -441.0865 & 0 & \#DIV/0! \\
\hline Fexx & 26 & 25 & -459.4651 & -601 & -24 \\
\hline Co & 27 & 26 & -477.8437 & 0 & \#DIV/0! \\
\hline \multirow[t]{11}{*}{$\mathrm{Ni}$} & 28 & 27 & -496.2223 & 0 & \#DIV/0! \\
\hline & 29 & 28 & -514.6009 & 0 & \#DIV/0! \\
\hline & 30 & 29 & -532.9795 & 0 & \#DIV/0! \\
\hline & 31 & 30 & -551.3581 & 0 & \#DIV/0! \\
\hline & 32 & 31 & -569.7367 & 0 & \#DIV/0! \\
\hline & 33 & 32 & -588.1154 & 0 & \#DIV/0! \\
\hline & 34 & 33 & -606.494 & 0 & \#DIV/0! \\
\hline & 35 & 34 & -624.8726 & 0 & \#DIV/0! \\
\hline & 36 & 35 & -643.2512 & 0 & \#DIV/0! \\
\hline & 37 & 36 & -661.6298 & 0 & \#DIV/0! \\
\hline & 38 & 37 & -680.0084 & 0 & \#DIV/0! \\
\hline
\end{tabular}




\begin{tabular}{|c|c|c|c|c|c|}
\hline & 39 & 38 & -698.387 & 0 & \#DIV/0! \\
\hline & 40 & 39 & -716.7656 & 0 & \#DIV/0! \\
\hline & 41 & 40 & -735.1442 & 0 & \#DIV/0! \\
\hline & 42 & 41 & -753.5228 & 0 & \#DIV/0! \\
\hline & 43 & 42 & -771.9014 & 0 & \#DIV/0! \\
\hline & 44 & 43 & -790.28 & 0 & \#DIV/0! \\
\hline & 45 & 44 & -808.6586 & 0 & \#DIV/0! \\
\hline & 46 & 45 & -827.0372 & 0 & \#DIV/0! \\
\hline & 47 & 46 & -845.4158 & 0 & \#DIV/0! \\
\hline & 48 & 47 & -863.7944 & 0 & \#DIV/0! \\
\hline & 49 & 48 & -882.173 & 0 & \#DIV/0! \\
\hline & 50 & 49 & -900.5516 & 0 & \#DIV/0! \\
\hline Sbxx & 51 & 50 & -918.9302 & -810 & 13 \\
\hline & 52 & 51 & -937.3088 & 0 & \#DIV/0! \\
\hline & 53 & 52 & -955.6874 & 0 & \#DIV/0! \\
\hline & 54 & 53 & -974.0661 & 0 & \#DIV/0! \\
\hline & 55 & 54 & -992.4447 & 0 & \#DIV/0! \\
\hline & 56 & 55 & -1010.823 & 0 & \#DIV/0! \\
\hline & 57 & 56 & -1029.202 & 0 & \#DIV/0! \\
\hline & 58 & 57 & -1047.58 & 0 & \#DIV/0! \\
\hline & 59 & 58 & -1065.959 & 0 & \#DIV/0! \\
\hline & 60 & 59 & -1084.338 & 0 & \#DIV/0! \\
\hline & 61 & 60 & -1102.716 & 0 & \#DIV/0! \\
\hline & 62 & 61 & -1121.095 & 0 & \#DIV/0! \\
\hline & 63 & 62 & -1139.473 & 0 & \#DIV/0! \\
\hline & 64 & 63 & -1157.852 & 0 & \#DIV/0! \\
\hline & 65 & 64 & -1176.231 & 0 & \#DIV/0! \\
\hline & 66 & 65 & -1194.609 & 0 & \#DIV/0! \\
\hline & 67 & 66 & -1212.988 & 0 & \#DIV/0! \\
\hline & 68 & 67 & -1231.367 & 0 & \#DIV/0! \\
\hline & 69 & 68 & -1249.745 & 0 & \#DIV/0! \\
\hline & 70 & 69 & -1268.124 & 0 & \#DIV/0! \\
\hline & 71 & 70 & -1286.502 & 0 & \#DIV/0! \\
\hline & 72 & 71 & -1304.881 & 0 & \#DIV/0! \\
\hline Taxx & 73 & 72 & -1323.26 & -1249 & 5 \\
\hline$W x x$ & 74 & 73 & -1341.638 & -1384 & -4 \\
\hline & 75 & 74 & -1360.017 & 0 & \#DIV/0! \\
\hline & 76 & 75 & -1378.395 & 0 & \#DIV/0! \\
\hline & 77 & 76 & -1396.774 & 0 & \#DIV/0! \\
\hline
\end{tabular}




\begin{tabular}{|c|c|c|c|c|c|}
\hline & 78 & 77 & -1415.153 & 0 & \#DIV/0! \\
\hline & 79 & 78 & -1433.531 & 0 & \#DIV/0! \\
\hline & 80 & 79 & -1451.91 & 0 & \#DIV/0! \\
\hline & 81 & 80 & -1470.288 & 0 & \#DIV/0! \\
\hline & 82 & 81 & -1488.667 & 0 & \#DIV/0! \\
\hline & 83 & 82 & -1507.046 & 0 & \#DIV/0! \\
\hline & 84 & 83 & -1525.424 & 0 & \#DIV/0! \\
\hline & 85 & 84 & -1543.803 & 0 & \#DIV/0! \\
\hline & 86 & 85 & -1562.181 & 0 & \#DIV/0! \\
\hline & 87 & 86 & -1580.56 & 0 & \#DIV/0! \\
\hline & 88 & 87 & -1598.939 & 0 & \#DIV/0! \\
\hline & 89 & 88 & -1617.317 & 0 & \#DIV/0! \\
\hline & 90 & 89 & -1635.696 & 0 & \#DIV/0! \\
\hline & 91 & 90 & -1654.074 & 0 & \#DIV/0! \\
\hline Uxx & 92 & 91 & -1672.453 & -1596 & 4 \\
\hline & 93 & 92 & -1690.832 & 0 & \#DIV/0! \\
\hline & 94 & 93 & -1709.21 & 0 & \#DIV/0! \\
\hline & 95 & 94 & -1727.589 & 0 & \#DIV/0! \\
\hline & 96 & 95 & -1745.967 & 0 & \#DIV/0! \\
\hline & 97 & 96 & -1764.346 & 0 & \#DIV/0! \\
\hline & 98 & 97 & -1782.725 & 0 & \#DIV/0! \\
\hline & 99 & 98 & -1801.103 & 0 & \#DIV/0! \\
\hline & 100 & 99 & -1819.482 & 0 & \#DIV/0! \\
\hline & 101 & 100 & -1837.86 & 0 & \#DIV/0! \\
\hline & 102 & 101 & -1856.239 & 0 & \#DIV/0! \\
\hline & 103 & 102 & -1874.618 & 0 & \#DIV/0! \\
\hline$X X$ & 104 & 103 & -1892.996 & 0 & \#DIV/0 \\
\hline
\end{tabular}

\section{References}

1. Journal of Advances in Physics Vol 12 No 4 page 4405 Multibody Energy States https://cirworld.com/index.php/jap/article/view/5965/5510

2. Handbook of Chemistry and Physics 2006-2007

3. Journal of Advances in Physics Vol 12 No 4 Magnetic Solution

4. Journal of Advances in Physics Vol 13 No 2 The Klein-Gordon Equation

\section{James W Goodman biography}

Over 50 hours of graduate physics and math courses.

BS physics

Followed the solution of the hydrogen Schroedinger equation.

Solved the hydrogen equation using group theory. 
Studied Hilbert Space and QM group theory.

Graded Hilbert Space group theory theorems.

89 percentile physics GRE

\section{Member of Mensa}

1971 The design of this digital cell phone is proprietary information for the Bell System.

1972 The Schoedinger equation was studied with the idea of writing down the potential energy between each pair of particles and adding them up.

1972 Two new assumptions were written down and from this the Schoedinger equation was solved. The electron electron interaction was solved exactly. The ground state energy of the first 10 elements was found exactly correct. New physics was found for the rest of the elements. The nuclear spin had been omitted.

1979 Bell needed a power forecasting and record keeping system. The secret was to use an exponential growth curve fitted to the three high values of the amperage. The statistical theory says that more users cause more mips cause more amps.

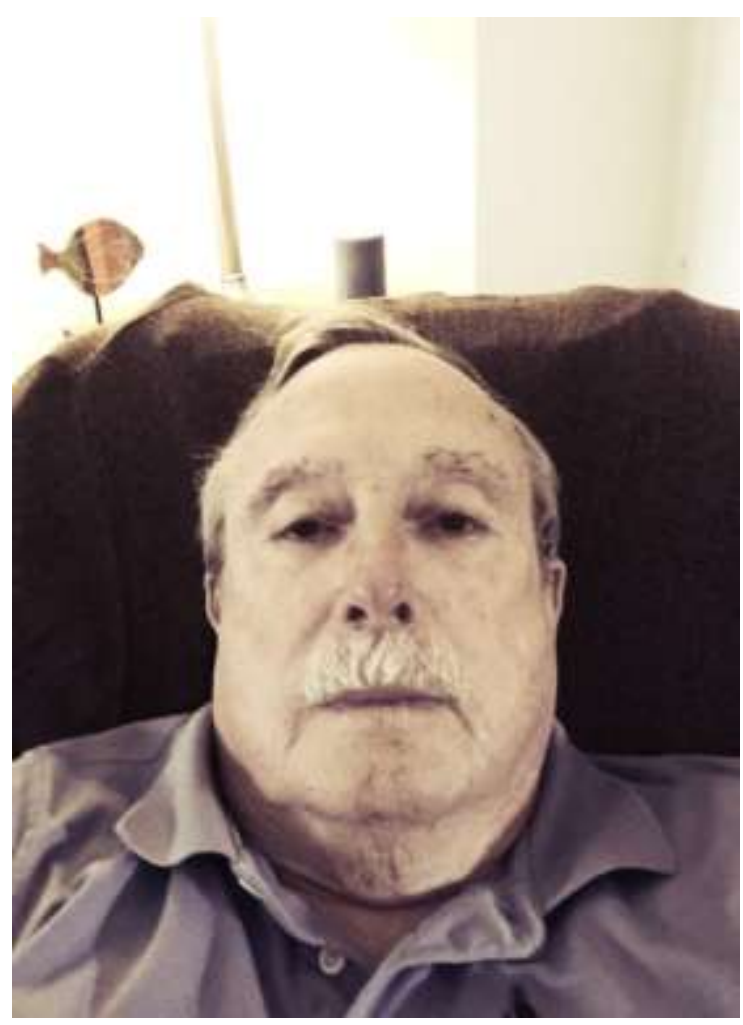

1980 's described the DSL to Bell and asked them to provide for students to hook up to the Internet. At that time the high-speed available was 2400 bits per second. DSL provided 1 million bits per second.

1980 's from study of the Aluminum gallium arsenide laser showed Cox cable how to turn around the repeaters. It shows that with the voltage below lasing the laser is a receiver.

1980 's from study of calculus of statistics and study of traffic engineering with the Bell System pointed out the problem with too many users on one T1 Line. At high usage after supper 10 users on one T-1 line would get about 100,000 bits per second. This increased the demand for DSL's.

1990's Suggested from a sawf study to Lockheed Martin the alloy LiAl for the skin of the shuttle tank. They used the Super Lightweight Tank for years. Each pound saved would put a pound added to the load in orbit.

2013 most reactions go through many energy states. Each difference in energy state represents gain or loss of a photon. The highest gain is the activation energy. The sum of the losses is the heat of formation. The greatest loss is the bond strength. 